\title{
IRRADIATION OF SPHERICAL TAREETS WITH MULTIPLE BEAMS AND HEMI-REFLECTORS
}

C. R. Phipps, Jr.

December 4 , to74

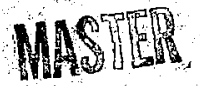

Prepared far U. S Atomic Energy Commission under contract No. W-7405-Eng-48

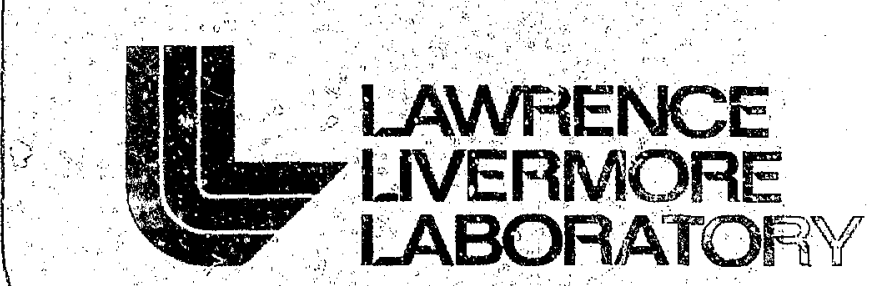

University of Calfomia/Livernore 


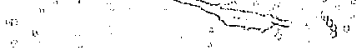

at

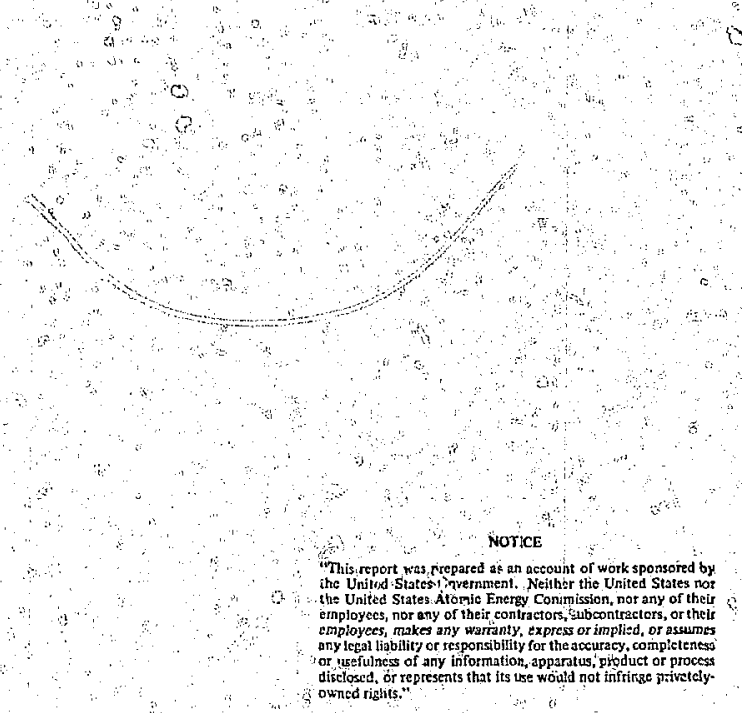

th

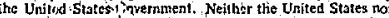
the United States Atonic Energy Conim ission, nat any of their employecs, makes any ryarsinty, exprass or inplited, or asesumas any lceal ligbility or tesponsibility for the sccuracy, compieteneso or lefefulncss af any ifformation, apparatus, pioduct or process diselosed, or tepresents that its use would not infrikge privetchy ouned rights.

\section{Printed in the United States of America Ávailable" from \\ National Technical Information Service \\ U.S, Department of Commerce 5285 Port Royal Road \\ Springfield, Virginia 22151 \\ Price: Printed Copy $\$$; Microfiche $\$ 1.45$}

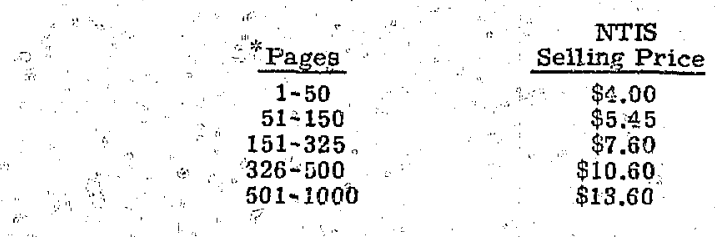


TID-4500, UC-34

Physics - General

\section{노 \\ LAWFENCE LNEAMOFE LABORATOFY

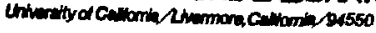

\section{UCRL-51706 \\ IRRADIATION OF SPHERICAL TARGETS WHH MULTIPLE BEAMS AND HEMI-REFLECTORS \\ C. R. Phipps, Jr.}

MS, date: December 4, 1974

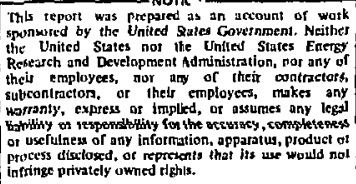

intringe privately owned dgh|s. 


\section{Contents}

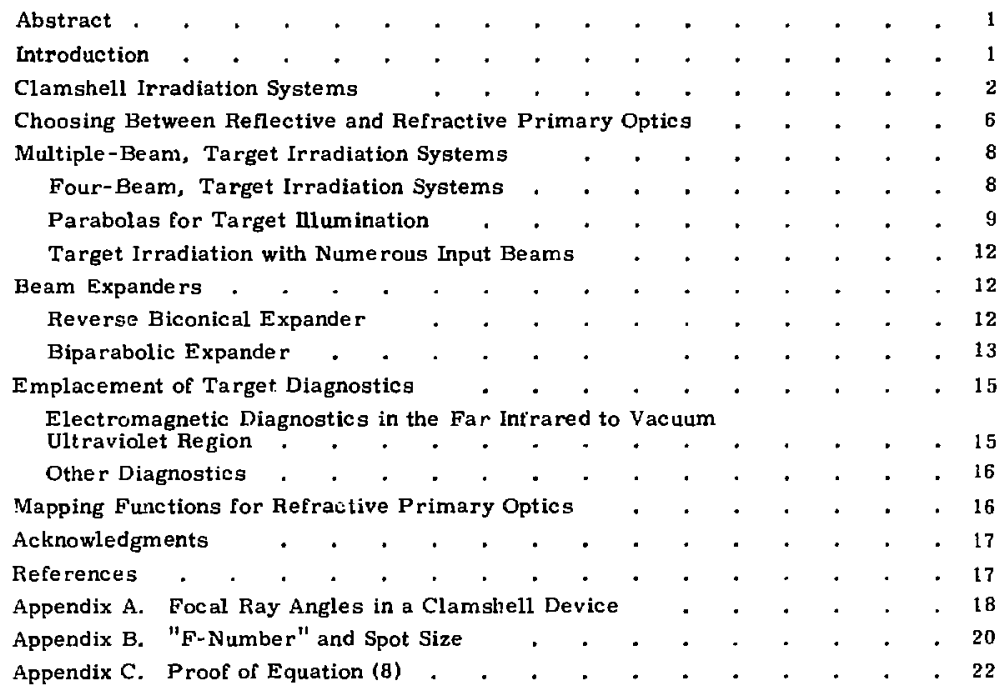




\title{
IRRADIATION OF SPHERICAL TARGETS WITH MULTIPLE BEAMS AND HEMI-REFLECTORS
}

\begin{abstract}
Approaches to the design of spherical irradiation facilities for laser fusion experiments are discussed. The purpose of such a facility is to focus multiple laser beams onto a spherical target, giving nearly total coverage and uniform intensity. It is assumed that each beam has a well defined phase and an intensity distribution which is cylindrically symmetric, but with an unspecified radial variation. The optical designs which are discussed have in common the ure of conic section reflectors to illuminate

the target. For each system discussed, mapping functions are derived which relate the dequirement for uniform irradiation of a sphere to a unique intensity distribution at the system entrance pupil. Means are described for combining the multiple laser beams to approximate the unique distribution of intensity required by the spherical irradiation system. Also discussed are the intessity mapping properties of beam expanding optics, and the impact of wrap-around optical systems on target diagnostics emplacement.
\end{abstract}

\section{Introduction}

As laser hardware is being developed, laser-plasma interaction experinents at LLL will progress from irradiating targets with 1 or 2 beams to expe riments employing perthaps 20 beams. The experiments now envisioned invol ve more or less uniform and surface-normal illumination of planar, cylindrical, and spherical targets. Methods of achieving planar and cyl indrical illumination a re well understood. This report deals with the pioblem of uaing a variable number of lase $r$ beams in a single, spherical, irr'adiation faciltty.

From an optical design polnt of view, the simplest such multibeam irradiation system is one in which individual focusing elements are centered in the faces of one of the five regular polyhedra, However, this approach has the following inherent disadvantages:

- The number of beams may only be 4, $6,8,12$, or 20 ; numbers that correspond to the number of faces of these five solids.

- Assuming cylindrically syrnmetric beams, the light projected onto a sphere will consist of a number of circular patterns. Tailoring of the overlap among these patterns to achieve near uniform intensity and near normal incidence around the sphere will involve complex apodization of the input beams, and extremely careful alignment. 
- A rather complex, numerically controlled, optic-positioning system operating in real time will likely be required to maintain optimum illumination of the target.

- Beam diagnosis is difficult.

There is an alternative type of irradiation system in which a single reflective structure maps essentially all vectors from the target location onto two or at most four entrance pupils. ${ }^{1-4}$ In this system multiple input beams are combined as concentric annuli, with simple apodization if the number of beams is small. Advantages of this approach are:

- The numier of beams may be any ever number in the case of two pupils, or any multiple of four for four pupils.

- The target irradiation can be tailored on a centimeter rather than a micron scali, and complicateu apodization is avoided.
- Maintenance of alignment requires onty colinearity of the input-beam symmetry axes.

- Real-time numerical control of target illumination is replaced by one-shot numerical control in machining the reflective structure.

Exanples of such reflective structures emplaying conic sections are described in Ref. 1, while illumination systems based in more general shapes are treated in Ref. 2. At the present state of development, the conic shapes are simpler to make and align, as well as adequate for the problem.

In the following sections, we discuss the relative advantages of the refractive versus reflective primary optics for this type of structure, and the means of achieving the required radial intensity distribution at the input pupil with single or multiple irput beams. We also discuss beam expanders, mapping functions, and the emplacement of target diagnostics.

\section{Clamshell Irradiation Systems}

Figure 1 shows a simple, 2-pupil, $4 \pi$ irradiation system consisting of two elliptical reflector sections as secondary optice and external, fast, aspheric lenses as primary optics. The ellipticel reflector sections are figures of revolution about the input beam axis, confocal at the target location, with eccentricity a $1 / 3$. With this eccentricity, the conjugate focus of each elliptical hemirellector is coincident with the surfacc of the upposite reflector. When the input or primary optics are refractive, the two foci conjugate to the target are real.

An optically equivalent design, in which these foci are virtual, substitutes internal, convex parabolic reflectors (shown by the dashed line) for the external refractive primaries shown by the solid line in Fig. 1. For this design, a unique, radial-intensity distribucion that results in uniform illumination of the target surface exists for the input beam and is given by ${ }^{1}$

$$
\frac{I(\rho)}{I_{0}}=\left[1+\left(\frac{\rho}{p}\right)^{2}\right]^{-2} .
$$

Here $I_{0}$ is a scaling constant for the input intensity and the other terms are defined in Fig. 1. If $\rho>\rho_{c}$, the collimated input beam will be scattered, and in this design $\rho_{c}=p$, the virtual focal length of the 


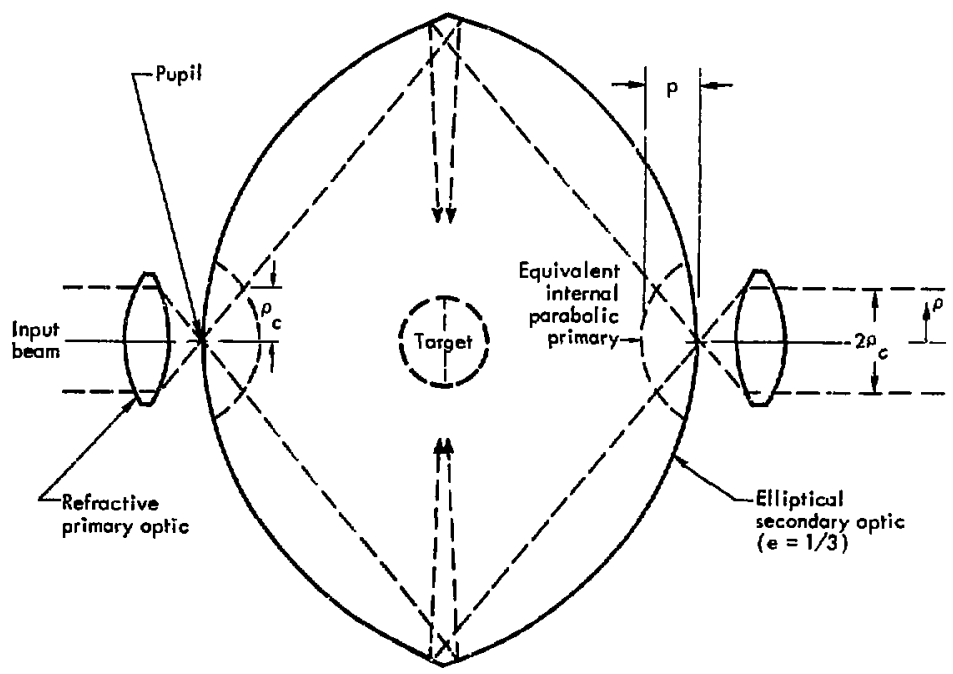

Fig. 1. Two-pupil, spherical illumination system with refractive primaries.

primary. Choosing $I_{0}=4 / \mathrm{p}^{2}$ gives unit intensity on the target-centered unit sphere.

Figures $2 a$ and $2 b$ show three input radial intensity distributions and the resulting angular distribution of radiation on the target surface. One of these is the "matched" distribution given by Eq. (1), which results in uniform target illumination. The two Gaussian inputs and their "outputs" on the target are included in Figs. $2 a$ and $2 b$ to demonstrato the moderate sensitivity of this mirror system to variations in the input beam profile. For econorny of laser energy, the matched input profile must be truncated or modified, since $50 \%$ of the total beam evergy lies outside $p=p_{c}$. This is done most easily by deliberate apodization, although the simple Gaussian shapes shown in Fig. 2 a reduce the wasted bean fraction to $31{ }^{\sigma}$. and $25 \%$, respectively, without affecting uniform lulumination of the target severely.

Designs employir'o rellective parabolic primary optics rejuire an entrance pupti of diameter $2 \rho_{c}$ for the collimated input beam. This is accomplished by slightly tilting the input beam and primary optic axes and deleting the entrance pupils from the elliptical secondary mirror. I slightly hyperbolic conves primary reflector, combined with an external collimating optic, is optically equivalent to a parabolic mirror, ${ }^{1}$ but is preferable to the parabolic primary. This combination preserves secondary mirror area by perinitting the entrance pupils to be reduced to pinholes, 


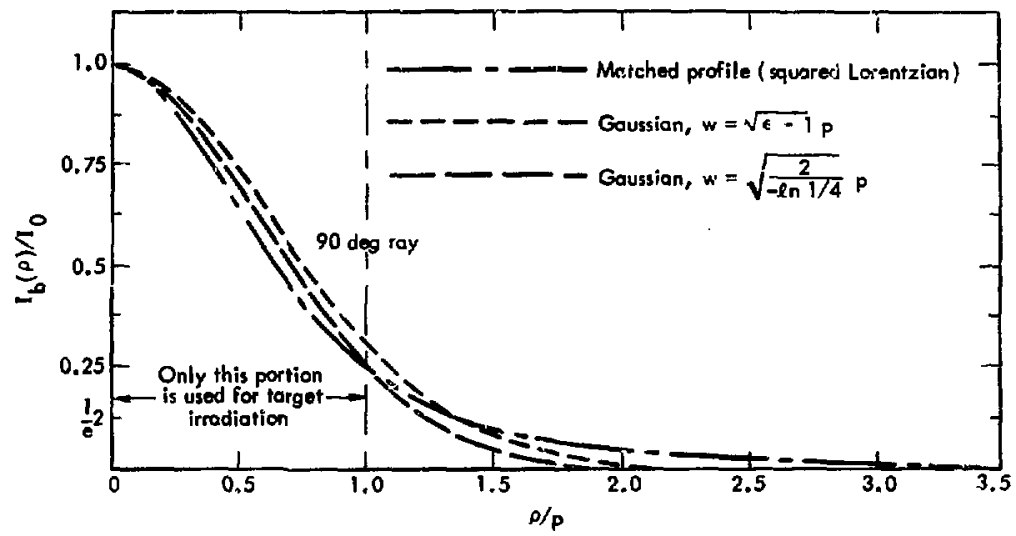

Fig. 2a. Radial intensity djst sibutions of input illumination in a clamshell device.

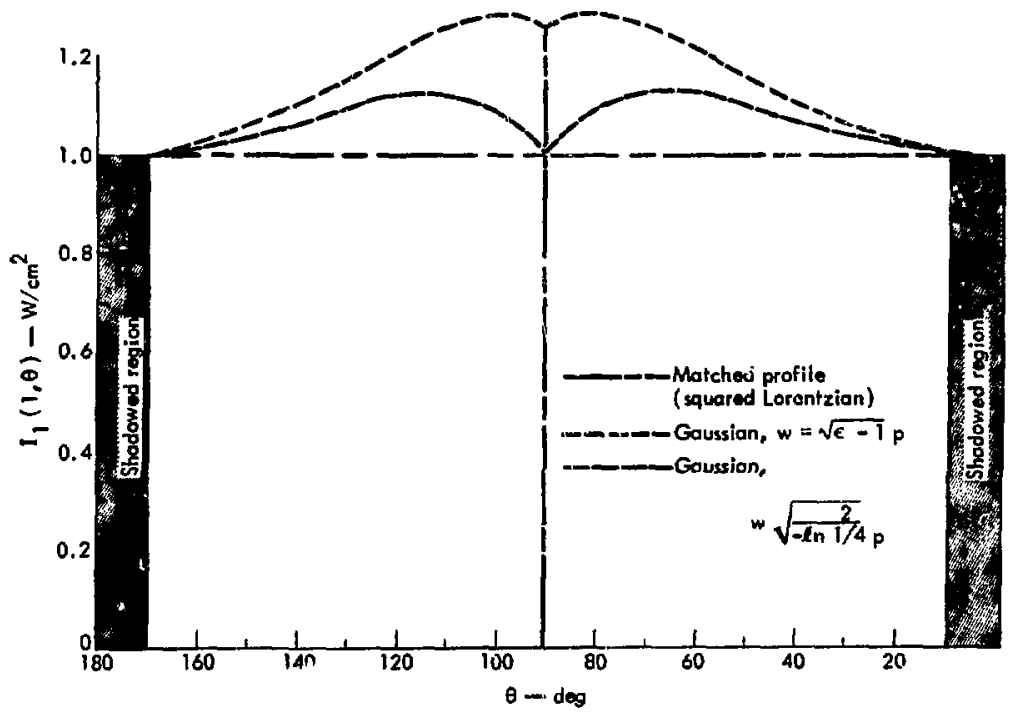

Fig. 2b. Angular intenusity distributions at the target in a clamshell device. 
which may be on- or off-axis, as shown in Figs, 3 and 4 . The collimating optic may be refractive or reflective, and is nornally a low speed device which requires only spherical surfaces. For example, the mirror systems illustrated in Figs. 3 and 4 illuminate $9 t \%$ of the target surface uniformly, and the collimating lenses shown have an f-number of 12.5 . Because of the optical equivalence, Eq. (1) still describes the illumination prc:erties of systems with hyperbolic primories.

With refractive primaries, each lens design will give a different intensity mapping function, and these functions are not generally calculable in closed for'm. However, uniform illumination of the target is assured if the angular istensity distribution at the real focus is given by $g_{2}(\phi)=4\left(1+3 \sin ^{2} \frac{\phi}{2}\right)^{-2}, 0 \leq \phi \leq 53.13^{\circ}$

where $\phi$ is the angle made by the input ray with the input axis. This, then, is the matching condition for refractive primaries. Equation (1) gives the correct input beam protile for uniform target illumination only if the input lens is optically equivalent to a parabolic reflector. To simplify discussion, it will be assumed that this is true for refractiv input optics featured in this report.

In any case, clamshell irradiation systems will involve the design of highly reflective, damage resistant surfaces. Since reflectivity depends upon the angle of incidence on metallic as well as multilayer dielectric surfaces, formulas for computing the incidence angles encountered in these systems are given in Appendix A.

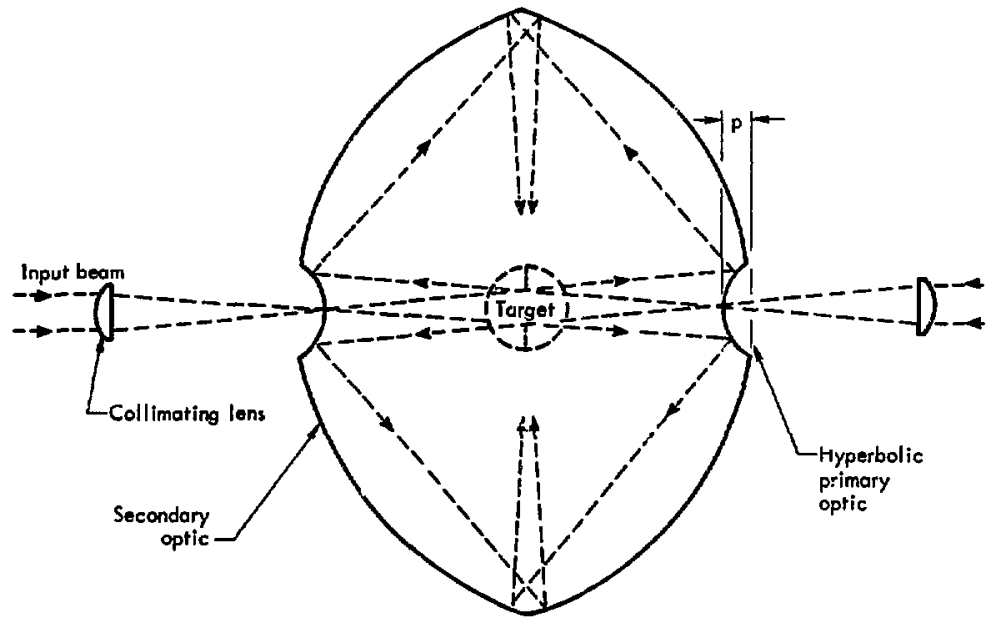

Fig. 3. Two pupil, spherical illumination system with normal reflective primaries. 


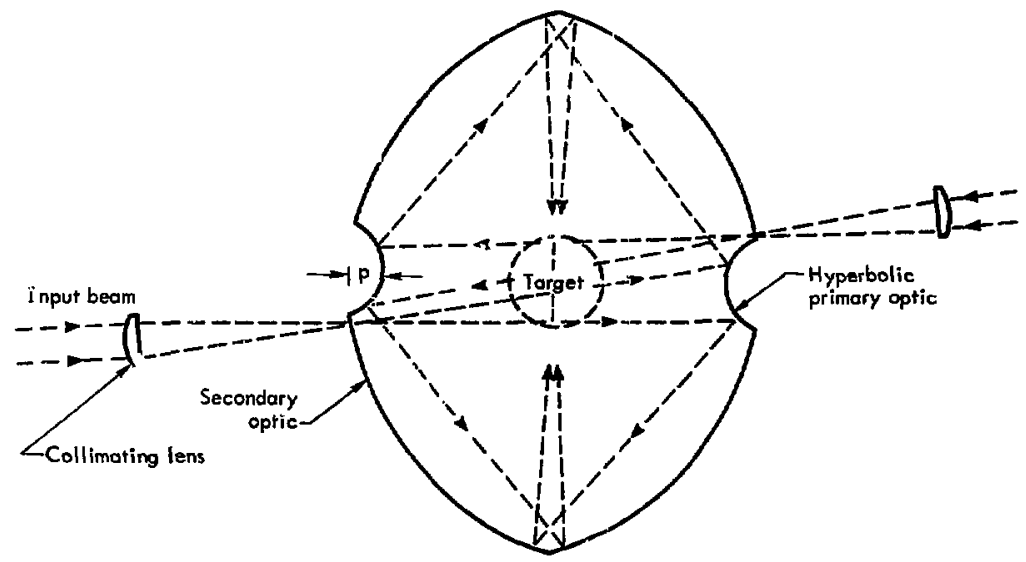

Fig. 4. Two pupil, spherical illumination system with thted reflective crimaries.

\section{Choosing Between Reflectiye and Refractive Primary Optics}

Reflective primary optics internal to the clamshell have certain advantages relative to external lenses, depending on irratiation energy level, diagnostic considerations, and other details.
The best approach for introducing laser energy to a clamshe'l-type irradiation system depents on the experi. mental circumstances (summarized in Table 1). 
Table 1. Comparis a of reflective and refractive primary designs.

\begin{tabular}{|c|c|}
\hline Configuration & Advantages \\
\hline $\begin{array}{l}\text { Normal refractive } \\
\text { primaries }\end{array}$ & 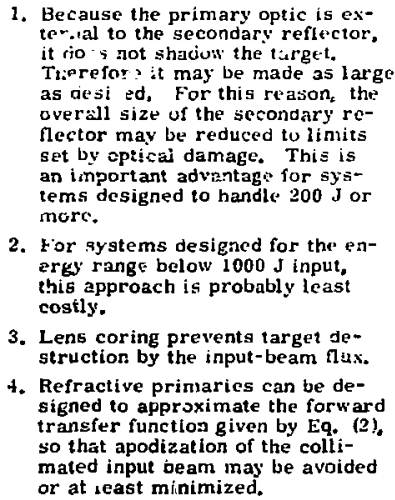 \\
\hline
\end{tabular}

Normal refective primarieb

Tuted reflective primaries
1. Aeflective systems are achromatic from the far IR into tho hard L' range, permitting the collection of wideband target diagnostic data at the entrance pupils. The large $f$-nLmber collimating lenses shown in Figs. 3 and 4 will not show severe chromatic aberration. If desired, these can be replaced by a collimating reflector without disturbint the illuniration symmetry, as would be the case for this gubstitution in Fig. 1.

2. Freedom from nonlinear refractive effects.

1. Freedom from chromatic aberrdfion.

2. Since the input beams do not cross the target region, delicate targets will not be damaged by input heam nux.

3. Freedom from nonlinear refractive effects.
1. This approach requires a iast lens, with a 106-deg full convergence angle (f-number 0.375). Such a lens, at present, must be cored to prevent internal vimage from lens surface ret etions. The core dous shadow the target to a degrec cumparable to the shadowing caused by reflective internal primaties, as in Figs, 3 and $t$.

2. Juch a lens cannot be actiromatic in a wideband sense, which complicates and probably prevents the colicction of target diagnostic data across the visible spectrum at the el.trance pupils.

3. Solid primaries are subject to nonlinear refractive effects at higl. power densities.

$i$, For systems designed for the $1000 \mathrm{~J}$ range or above, the primary lens becomss larga and costly, so that it is not inimediately obvious that al -reflective systens cost mort.

1. Delicate targets may be destruyed by the input-beam fiux, which will be of the order of $10 \mathrm{~J} / \mathrm{cm}^{2}$ in the target region, in early evperiments.

2. This approach probably :nvives mare cost for systems designed for tine $1000 \mathrm{~J}$ input range or below.

3. Reflective primarias tefinitely imply input-beam apodizations.

1. Slight asymmetry in tho target irradiation distribution due to tilting of the primary cpics.

2. This approach probably invclves more cost for systems designed for the $1000 \mathrm{~J}$ input range or below.

3. Reflective primaries defititely imply input beam apodization. 


\section{Multiple-Beam, Target Irradiation Systems}

FOUR-BEAM, TARGET IRRADIATION SYSTEMS

In going from two to four input beams, the main task is to combine input beams in such a way as to preserve uniform tariget illumination with minimum complexity and cost.

The first and most obvious way of doing this with four equal energy beams is shown in Fig. 5. Each hemi-reflector is divided into zones subtending equal solid angles at the target by 60-deg half-angle cones. At each of the two input pupils, these zones map into an outer annulus (beams 2 and 3) surrounding a "core" beam (beams 1 and 4) which occupies the central $58 \%$ of the pupil diameter (see Fig. 5). The combined entrance pupil radial-intensity distribution must still approximate the matched input (see Fig. 2) to achieve uniform target illumination. Deliberate attention must be paid to obtaining the approprlate amplified beam profiles by means of apodization at the oscillator level to avoid wasting laser energy. The annular beam may be derived from an unstable resonator configuralion, or from a solid beam passed through a Cassegrainian expander.

An alternative approach has been suggested for combining four beams in a single irradiation facility ${ }^{4}$ (Fig. 6). Surprisingly the optical transfer properties of this design are almost identical to those of the design shown in Fig. 5. In this alternative approach, the core beams (1 and 4) are transferred to the target by a partiai clamshell secondary system,

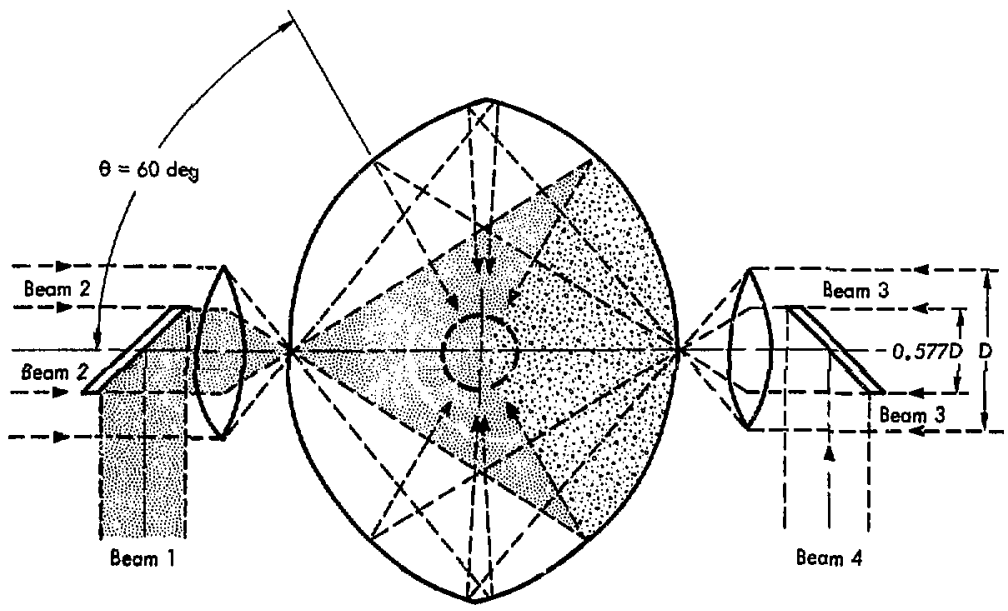

Fig. 5, Fuur-beam irradiation system employing clamshell secondary optics. 


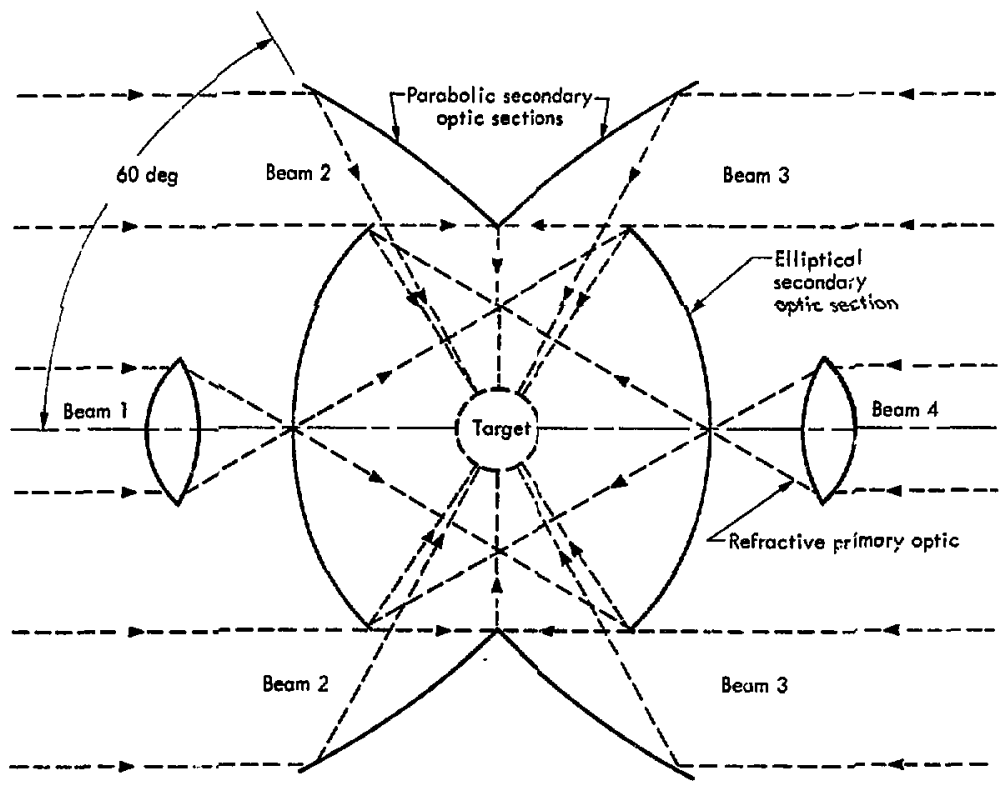

Fig. 6. Four-beam irradiation system employing elliptical and parabolic secondary optics.

while the annular beams ( 2 and 3 ) are traisferred by the parabolic secondary reflectors shown in the figure.

An advantage of this system is that the use of refractive primaries for beams 1 and 4 is madc more practical, since now the full convergence angle required from these lenses is only 64.4 versus $106.3 \mathrm{deg}$ (f-number 0.794 vs $f$-number $0.375^{*}$ ) for the previous design. On the minus side, larger and more expensive input optics are needed (beam expanders for example), and the secondary aptic system acw con-

\footnotetext{
"See Appendix $\mathrm{h}$.
}

tains 4 rather than 2 individual elements uhich mus: be aligned with the target. Since no further line-of-sight access to the target is provided by this design than the one shown in Fig. 5, this design would seem to offer few advantages.

\section{PARABOLAS FOR TARGET ILL UMINATION}

Both for purposes of direct target illumination and for designing Cassegrainian expanders, we will need to know the mapping function of a simple parabolic reflector (see Fig. 7). 


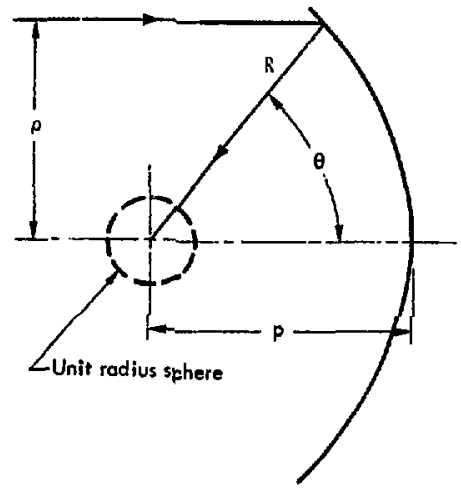

Fig. 7. Definition of terms used in the discussion of parabolic reflector mapping functions.

The equation of the parabolic surface is

$$
R(\theta)=p \sec ^{2} \frac{\theta}{2} .
$$

By analogy, ${ }^{1}$ the mapping onto a focalcentric unit-radius sphere is

$f(\theta)=\frac{I_{1}(I, \theta)}{I_{b}(\rho)}=R^{2}(\theta)=p^{2} \sec ^{4} \frac{\theta}{2}$.

This function is plotted in Fig. 8 ,

To be comparable with the analysis in Ref. 1, the reverse mapping function will be derived by converting to radial coordinates and inverting:

$$
\rho(\theta)=R(\theta) \sin \theta .
$$

Substituting into Eq. (3),

$$
\rho(\theta)=2 p \tan \frac{\theta}{2} \text {. }
$$

Equations (4) and (5) may now be combined to give the reverse mapping function, which is the necessary input beam intengity

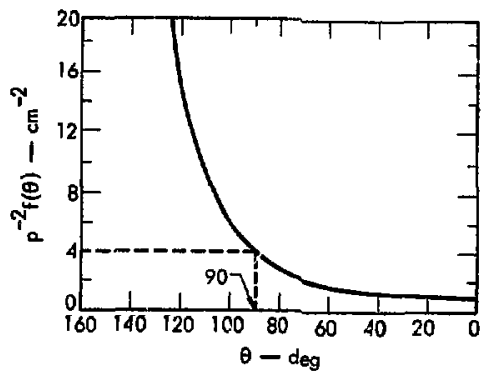

Fig. 8. Forward mapping function for a parabolic reflector.

profile for uniform targe illumination with this system:

$\frac{I(\rho)}{I_{0}}=g\left(\frac{\rho}{p}\right)=f^{-1}=\frac{1}{p^{2}}\left[1+\left(\frac{p}{2 p}\right)^{2}\right]^{-2}$.

This function is plotted in Fig. 9.

The only differences between this matched input for a parabolic reflector and that for a clamshell sytstem (see Eq. (1)) are as follows:

- Scaling - The peak value for the transfer function is only 1/4 that for the clamshell system.

- Approximation - For target illumination purposes, the tail rather than the center of the transfer function is used. By referring to Fig. 2, it will be seen that it is not clear that Gaussian apodization will give a good fit to the desired function. In fact, if either end point of the matched input section shown in Fig. 9 is matched by a Gaussian, the error at the other end point of the section will be at least $59 \%$. By contrast, a simple linear approximation matching both end points is in error by no nore than $26 \%$, at $\rho / p=2.64$. 


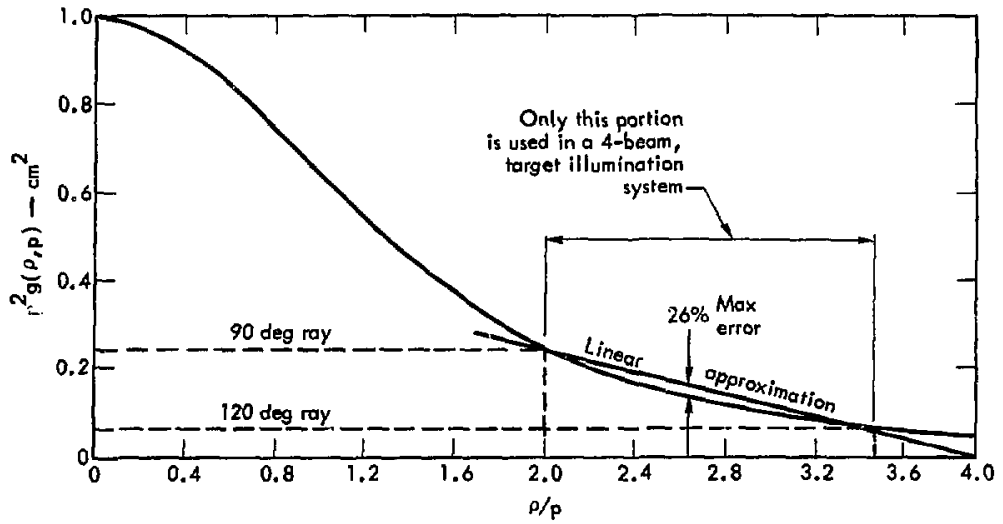

Fig. 9. Reverse mapping function for a parabolic reflector.

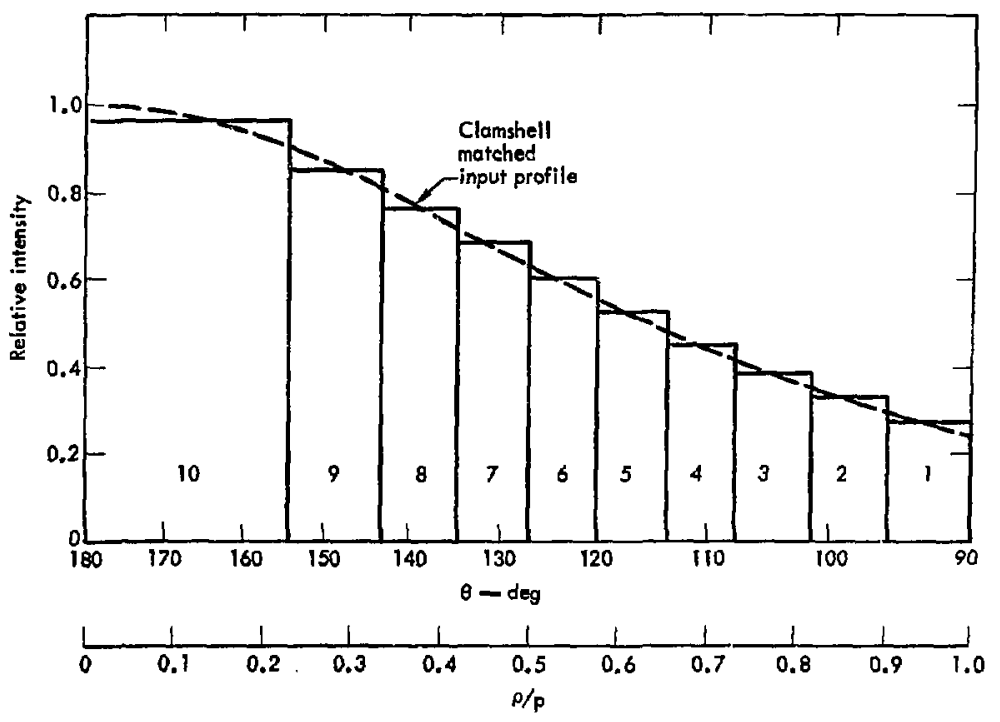

Fig, 10, Annular beam stacking in a 20-beam irradiation system. 
However, apodization is likely to be required to achieve any specific functional form of input distrusution, so that a study of functional approximations to the matched input would be of little value.

TARGET IRRADIATION WITH NUMEROUS INPUT BEAMS

As the number of input beams becomes large, there is a clear advantage to combining them in an annular fashion at the entrance pupils of a simple clamshell irradiation system. Otherwise, the advantages of a monolithic irradiation system relative to a system with multiple lenses begin to vanish. The reason for this becomes clear by referring to fig. 10, which shows the input intensity distribution profile at one input pupil of a clamshell irradiation system employing 20 beams of equal energy. With no effort to smooth the transitions between beams, the error is never more than about $5 \%$ relative to the desired input profile. Transition radii are computed from the expression $\frac{\rho_{m}}{p}=2 \tan \left\{\frac{1}{2} \arccos \left[\frac{3+(5 m / N)}{5+(3 m / N)}\right]\right\}$

for the $m$ th transition in a $2 \mathrm{~N}$ beam system, assuming equal energy in the $2 \mathrm{~N}$ beams. The required input beam intensity in the $\mathrm{mth}$ annulus to achieve uniform target illumination is computed from liq. (1). Since a 20-beam facility is a definite possibility at LIL, the relevant parameters are listed in Table 2.

Table 2. Parameters for a 20-beam system (see Fig, 10).

\begin{tabular}{cccc}
\hline $\begin{array}{c}\text { Interface } \\
\mathrm{m}\end{array}$ & \multicolumn{1}{c}{$\begin{array}{c}\theta_{\mathrm{m},} \\
\mathrm{deg}\end{array}$} & $\frac{1\left(\rho_{\mathrm{m}}\right)}{\mathrm{I}_{0}}$ \\
\hline 0 & 1.0000 & 90 & 0.2500 \\
1 & 0.9045 & 95.74 & 0.3025 \\
2 & 0.8165 & 101.54 & 0.3600 \\
3 & 0.7338 & 107.46 & 0.4225 \\
4 & 0.6547 & 113.58 & 0.4900 \\
5 & 0.5774 & 120 & 0.5625 \\
6 & 0.5006 & 126.87 & 0.6400 \\
7 & 0.4201 & 134.43 & 0.7225 \\
$\mathrm{~B}$ & 0.3333 & 143.13 & 0.8100 \\
9 & 0.2294 & 154.16 & 0.9025 \\
10 & 0.0000 & 180 & 1.0000 \\
\hline
\end{tabular}

\section{Beam Expanders}

Beam expanding optics will be required to generate the annular beam profiles which are needed in a multiple-beam irradiation system. We will limit discussion to two types of reflective Cassegrainian devices that have useful optical mapping properties, the biconical and the biparabolic expanders. The first is simple to build and possesses a gently varying mapping function which might be useful in beam shaping. The second is more expensive but has the advantage of preserving the input-beam profile.

\section{HEVERSI: RICONICAL LXPAXIIR}

Figure 11 shows the reflective wurface cross section, which is a figure of rotation about tho input bosm axis, for this device. It cam be shown that, for in arbitrary cone having haif-angle 3 (aes: Appendix C), 


$$
d \boldsymbol{r}+d \rho=0 .
$$

Therefore, this device expands or contracts an annular beam of light without changing its thickness. The forward transfer funcuon for the beam expander is

$$
i(x, \rho)=\frac{I(r)}{I(\rho)}=-\frac{\rho d \rho}{r d r}=\frac{\rho}{r} .
$$

But, from Eq. \{8\},

$$
r+p=c \text {. }
$$

So.

$$
f(r)=\left(\frac{c}{r}-1\right) .
$$

while

$$
g(p)=\left(\frac{c}{\rho}-1\right) .
$$

The mapping functions are plotted in Figs, 12 and 13 for an expander with peripheral magnification $M=c / a: 2$. It is easily shown that the analogues to Eqs. (8), (11), and (12) for the forward geometry are, respectively:

$$
\begin{aligned}
& d r-d p=0, \\
& f(r)=\left(1-\frac{b}{r}\right) .
\end{aligned}
$$

and

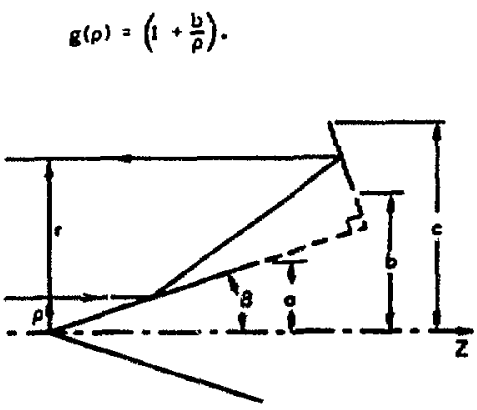

Fig. II. Keverac biconical expander.
These functions are increasing rather than decreasing functions of radius.

\section{BIPARABOLIC EXPANDER}

The expander illustrated in Fig. 14 consists of two parabolic reflectors sharing an axis of symmetry and oriented in the same direrition.

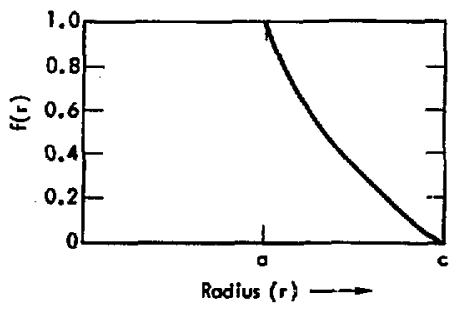

Fig. 12. Forward mapping function for a $2 \times$ biconical expandt:.

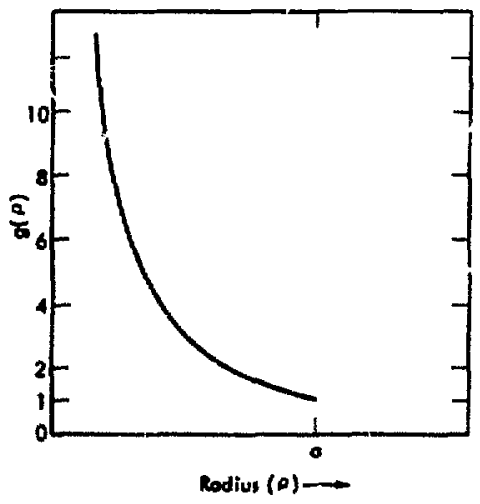

Nig. 13. Reverae mapping function ro* * $2 \times$ biconical expantler. 
Since

$$
\begin{aligned}
& R_{1,2}(\phi)=p_{1,2} \sec ^{2} \frac{\Phi}{2}, \\
& \rho=2 p_{1} \tan \frac{\phi}{2},
\end{aligned}
$$

and

$$
r=2 p_{2} \tan \frac{\Phi}{2}
$$

the linear magnification of the device is given by

$$
\mathbf{M}=\frac{\mathbf{r}}{\rho}=\frac{\mathbf{p}_{2}}{\mathbf{p}_{1}} .
$$

Also, it follows immediately that

$$
d r=M d \rho,
$$

and

$$
f(r)=\left(\frac{R_{1}}{R_{2}}\right)^{2}=M^{-2}=[g(\rho)]^{-1}
$$

for all values of $\mathbf{r}$ and $p$.

This type of expander is then useful for preserving beam intensity distributions during uniform linear magnification.

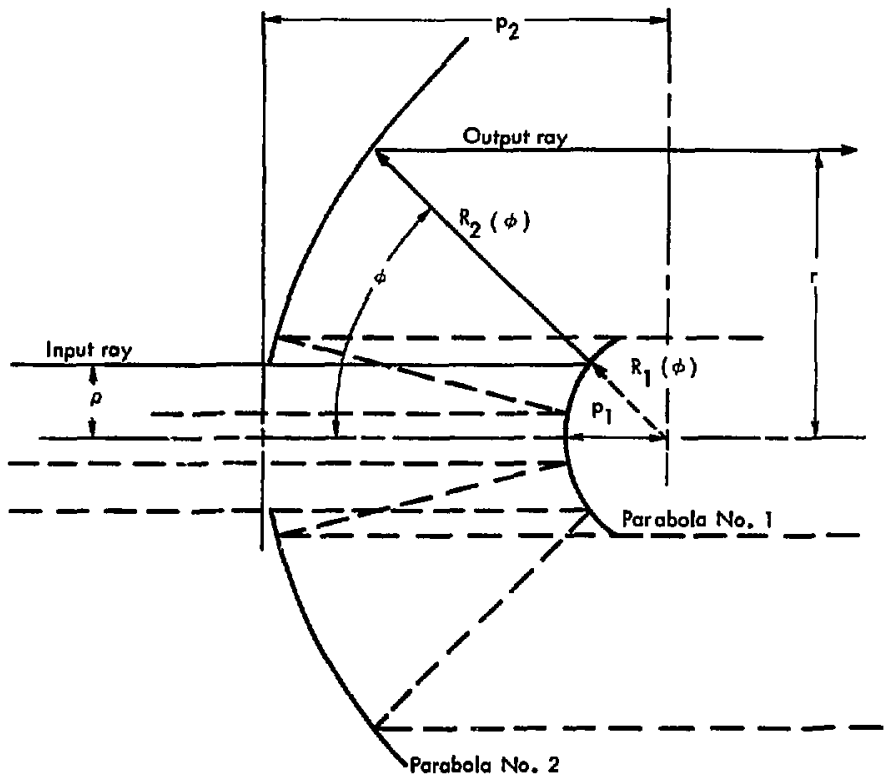

Fig. 14. Biparabolic expander. 


\section{Emplacement of Target Diagnostics}

ELECTROMAGNETIC DIAGNOSTICS IN THE FAR INFRARED TO VACUUM ULTRAVIOLET REGION

All of the spherical irradiation systems we have discussed are characterized by essentially single-valued and analytic mapping in the sense that:

- Each direction from the target focus that is accessible to the device is mapped in a one-to-one fashion onto a point in the input pupil (s), and vice versa.

- Those regions of the mapping which are singular are not accessible for practical reasons.

Those systems which use reflective optics exclusively also have the following desirable physical properties:

- Complete freedom from comatic. chromatic, spherical, and astigmatic aberrations, as well as field curvature, for incorning plane waves.

- Nearly complete freedom from the off-axis aberrations for outgoing incoherent radiation due to the extremely small ratio (of order $10^{-4}$ ) of target to reflector dimensions obtained,

- Invariant transit time (for spherical targets) between the target surface and the input pupil(s).

- Extremely broad bandwidth, IImited at long wavelengths by the diameter of the input pupil(s) and at short wavelengths by reflector sibsorptivity. In addition, all systems are characterized by:

- Maximum opticel speed. This means that the focal-region size in these systems is minimally sensitive to input beam divergence relative to that produced by annther syst $2 \mathrm{~m}$ having the same total illumination solid angle, but multiple, Ioncontiguous focusing components, 'or plane wave inputs. Also these systems offer maximally efficient collection of target radiation, permitting total calorimetry of reradiated energy.

- Simple mapping. This means that the direction in which radiation originates from the target focus can be unfolded in a simple manner at the input pupils. It should be noted that two further advantages are possessed by systems such as those shown in Figs, 1, 3, 4, and 5 . Systems which have a real focus conjugate to the target permit placement of a field stop at the conjugate focus. The mapping which results is now truly single-valued for incoming or outgoing radiation, in the sense that each point on the target surface which is accessible is mapped one-to-one onto a point in the input pupil(s). In addition, the off-axis aberrations disappear.

Considering these properties, a significant number of the usual target diagnostic requirements which customarily involve the deletion of input illumination solid angle can be satisfied simultaneously (with beamsplitters) at the entrance pupil(s) of the system we have described. In the far infrared (FIN) to vacuum ultraviolet (VUV) band these include:

- Spectrometry, withont loss of directional information.

- Calorimetry, without loss oi directional information.

- Discrimination between coherent and incoherent target radiation, by means 
of opening and closing the field stop in systems with real conjugate foci.

- Interferometry.

- Isobar velocity measurement over a wide range of density.

- Measurement of collective plasma phenomena by light scattering.

- Measurement of self generated magnetic fields by Faraday rotation.

- Streak photography.

\section{OTHER DIAGNOSTICS}

Mezsurement of the flux, mass, velocity, and charge of particles emitted by the target as well as $x$-ray spectrometry and interferometry, calorimetry, and target handling must be done through apertures in the secondary reflector(s).
A system which provides essentially $4 \pi$ illumination offers no line-of-sight access to the target, except by reflection. However, the solid angle required for these activities need not be large to permit effective measurements. This is especially true since such systems will be used in experiments intended to produce large fluxes of energetic particles and photons. Finally, diagnostic apertures for fast particles and hard photons would not be impaired by a thin reflective foil covering which doubles as a portion of the secondary reflector. It is unclear whether those diagnostic apertures which require direct access to the target should be arranged in a thin annulus in the target equatorial plane or randomly around the secondary mirror.

\section{Mapping Functions for Refractive Primary Optics}

For definitude, we have considered refractive primary optics which are equivalent to parabolic reflectors in this report, since simple analytic solutions for this case exist. However, refractive optics offer considerable nexibility in matching laser radial-intensity dictributions to the angular input distribution required by clamshell irradiation systems (F.q. 2). This is because the value of the refractive irilex, $n$, determires the overall behavior of the forward intensity mapping function, $\ell(\phi)$, of a wide-angle diffraction-limited lens.

To Lllustrate this fact, the mapping functions of two computer-designed diftraction-limitcd lenses were calculated with the assigtance of Anne Grcenbaum of 1.LL. Both lenses were designed to iluminate the $106.3 \mathrm{deg}$, full angle required by hemi-reflector systems. The code calculated intensity versus incidence angle $\phi$, at the lens focus from $\phi=0$ (on axis) to $\phi=53 \mathrm{deg}$. The first lena with $n=1.50$ mapped uniform input intensity into a function which decreased from its on axis value of unity to $\sim 0.75$ at $\phi=40$ deg. The second lens with $n=4$ gave a mapping function which inc reased steeply with $\phi$ to a value of 5.8 at $53 \mathrm{deg}$.

The reason for this behavior can be seen from the following heuristic argument. We have shown that the mapping function of the parabolic reflector is an increasing function of angle at the focus (see Fig. 8), This is because the radlus of curvature of the mirror is always greater than its focal length, so that a 
tangential bundle of rays sees a greater intensification due to the inverse-square law than an axial bundle. However, the front surface radius for a glass lens is always smaller than the focal length, leading to the opposite result. For paraxial rays, the front surface radius of a plano-convex lens is given by $R_{L}=$ (n-1), while that of a parabolic mirror is given by $\mathbf{R}_{M}=2 f$. Therefore, as the lens reIractive index $n$ is continuously increased Irom, 1.5 to 4.0, its forward mapping functiou also changes continuously from a decreasing to an increasing function of focal incidence angle, passing through the parabolic mirror case in an approximate sense.

\section{Acknowledgments}

It is a pleasure to acknowledge the encouragement and useful suggestions of Dr. Hal Ahlstrom. In addition, the author gained valuable understanding of the proper- ties of diffraction-limited lens designs from several discussions with Dr. John Hunt and from numerical, intensity-distribution calculations performed by Ms. Anne Greenbaum.

\section{References}

1. C. R. Phipps, S. E. Bodner, and J. W. Shearer, A Reflective Optics System for Uniform Spherical Ilumination, Lawrence Livermore Laboratory, Rept. UCRL75626 (1974).

2. G. Minerbo, An Optical System for Laser Fusion, Los Alamos Scientific Laboratory, Rept. IA-UR 74-604 (1974).

3. J. Tillotson, KMS Industries, Inc., private communication (1974).

4. H. Ahlstrom and J. Nuckolls, Lawrence Livermore Laboratory, private communication (1974).

$\mathrm{HLL} / \mathrm{bl} / \mathrm{lmc}$ 


\section{Appendix A. \\ Focal Ray Angles in a Clamshell Device}

In discussing clamshell-type irradiation systems, one frequently encounters the problem of transforming from angular to radial coordinates when computing the ray incidence angle at the elliptical surface, or calculating the required input convergence angle. From Ref. 1, we list here the formulae which are used it: these conversions, specialized to :te case $e_{1}=1 / 3$. Figure Al defines the terms used in this appendix and shows the geometrical relationships pertaining to one half of a completely general clamshell system with $e_{2} \geq 1$. In particular, $\phi$ is the convergence half angle at either primary focus, $\theta$ is the incidence angle on the target focus, $\frac{\gamma}{2}$ the incidence angle on the elliptical reflector and $\rho$ is the radial coordinate of an input ray prirr to focusing on the clamshell input pupil. The incidence angle on an internal reflective primary optic depends on the specific configuration. This angle $\mu$ is gi ren by ${ }^{1}$

$$
\mu=\frac{\phi}{2}+\psi=\frac{\phi}{2}+2 \arctan \left[\left(\frac{e_{2}-1}{\mathrm{e}_{2}+1}\right) \tan \frac{\phi}{2}\right] \text {. }
$$

The radial coordinate in the collimated input beam is given by

$$
\rho=2 p \tan \frac{\phi}{2} \text {. }
$$

The relationship between the angles $\phi$ and $\theta$ is

$$
\Phi=2 \arctan \left(\frac{1}{2} \cot \frac{\theta}{2}\right) .
$$

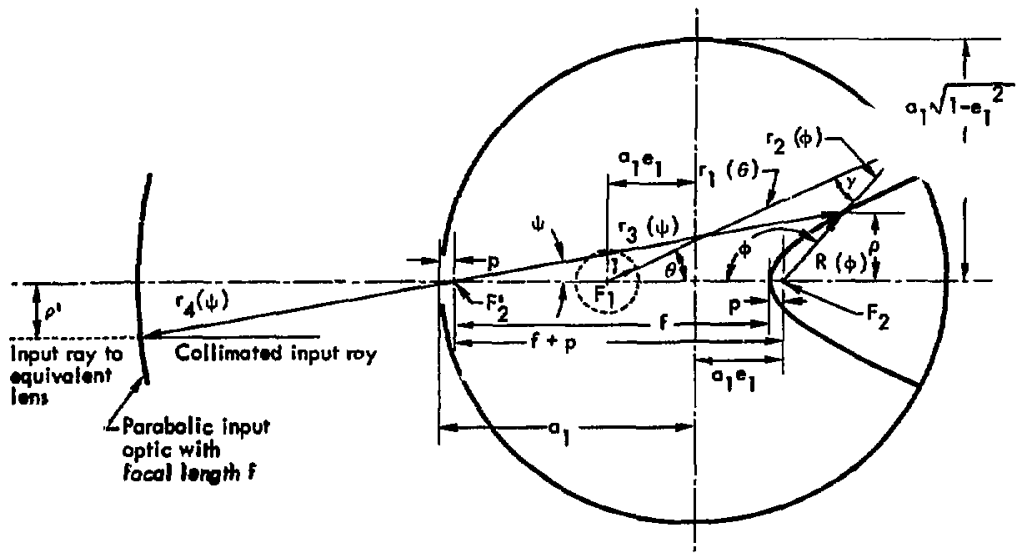

Fig. A1. One-beam illumination system with hyperbolic primary and parabolic input reflector: 
From this result, Eq. (A2) becomes

$$
\left(\frac{\rho}{p}\right)=\cot \frac{\theta}{2} \text {. }
$$

l'inally,

$$
\gamma=\pi-\{\theta+\phi) .
$$

These parameters also apply to an equivalent external refractive primary optic with focus at $\mathrm{F}_{2}$. These last three expressions are plotted in Fig. A2.

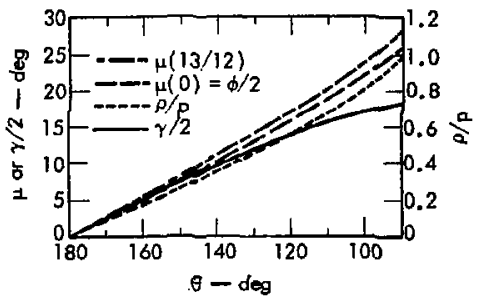

Fig. A2. Dependence of $\phi, \rho, \gamma$, and $\mu$ on $\theta$ in a two-beam illumination system. 


\section{Appendiz B. \\ 'F-Number' and Spot Size}

The $f$-number of a focusing optic is the matching parameter between optical elements as well as a determining factor in diffraction-limited spot-size calculations. There are two definitions of this quantity, one which is customary and one which is generalizable to $4 \pi$ iriadiation while at the same time reducing to the customary form at small solid angles of illumination.

The customary definition is

$$
f_{\#}=f / D=(2 \tan \phi)^{-1} \text {. }
$$

where $D$ is the pupil diameter of the optic and $f$ its focal lengtb. Phi is the halfcone angle made by the extreme rays passed by the pupil. This definition is used throughout this report for convenience only in describing refractive primary optic elements.

The customary definition runs into difficulty when $\phi$ approaches $90^{\circ}$. Here a secondary definition is useful; it is completely general, as well as being tied directly to the convergence solid angle of the optic, permitting quick estimation of spot size from etendue considerations ${ }^{\text {** }}$ (cf. the antenna theorean). The limiting solid angle illuminated by a large fnumber optic, according to the customary definition of the $\mathrm{f}$-number, is given by:

$$
\Delta \Omega=\frac{\pi}{4 \mathrm{f}^{2}}
$$

Therefore,

\footnotetext{
"See P. Jacquinot, Rep. Prog. Phys,
} 23. $270(1960)$.
$f_{H}=[8(1-\cos \phi)]^{-1 / 2}=\left(\frac{\pi}{4 \Omega}\right)^{1 / 2}$

is a completely general definition which approaches the customary one asymptot. ically at small values of the convergence half angle, $\phi$. Under this definition, the f number of a $4 \pi$ irradiation system is 0.25 , or that of half a clamshell is $\mathbf{0 . 3 5 4}$. Similarly, for the two lenses discussed in the section Four-Beam Target Irradiation Systems, we hrve:

\begin{tabular}{lcc}
$\phi$, deg & Customary $f_{\#}$ & General $f_{\#}$ \\
\hline 53.2 & 0.375 & 0.559 \\
32.2 & 0.794 & 0.901
\end{tabular}

This distinction becomes important when diffraction-limite's, spot size is to be estimated, given the intensity profile of the collimated input to a focusing optic. Etendue $\Phi(Z)$ is related to the Poynting vector $\mathrm{S}$ by

$\Phi(z)=\frac{1}{S_{0}} \int S\left(x, y, x, \frac{\Lambda}{k}\right) d \times d y d^{3} \hat{k}=\lambda^{2}$.

The etendu theorem, which derives either from the antenna theorein or fron. thermodynamics, states that etendue is conserved between any two-beam cross sections in a lossless optical system. This fairly obvious statement becomss more useful when expressed in terms of the effective area and solid angle in the collimated region of $a$ beam ( $k$ ) and in the focal region (o):

$$
\Phi=A_{b} \Omega_{b}=A_{0} \Omega_{0}=\lambda^{2} .
$$


Table B1. Properties of Gaussian and circ furctions.

\begin{tabular}{|c|c|c|c|c|c|}
\hline Function & $\begin{array}{c}\text { Radial } \\
\text { near field } \\
\text { distribution }\end{array}$ & $\begin{array}{c}\text { Angular } \\
\text { far field } \\
\text { distribution }\end{array}$ & $\begin{array}{l}\text { Collimated } \\
\text { cross-section } \\
\text { "asea" } \mathrm{A}_{\mathrm{b}}\end{array}$ & $\phi_{\mathrm{b}}$ & $\begin{array}{l}\text { Beam etendue } \\
{ }^{{ }_{\mathrm{b}}} \cong \mathrm{A}_{\mathrm{b}} \pi \phi_{\mathrm{b}}^{2}\end{array}$ \\
\hline Circ & $\operatorname{circ}\left(\frac{\rho}{w}\right)$ & {$\left[\frac{J_{1}(2 \phi / \Psi)}{(2 \Phi / \Psi)}\right]^{2}$} & $\pi w^{2}$ & $1.292 \cdot \frac{\lambda}{\pi w}$ & $1.67 \lambda^{2}$ \\
\hline Gaussian & $e^{-2(p / v)^{2}}$ & $e^{-2(\phi / \Psi)^{2}}$ & $\pi w^{2}$ & $1.000 \frac{\lambda}{\pi \mathrm{W}}$ & $\lambda^{2}$ \\
\hline
\end{tabular}

The details depend on the radial intensity profile of the collimated beam and the corresponding angular distribution at the focus. Two are shown in Table BI. In this table, the near and far fieid distributions are related by Fourier transformation. $\operatorname{Circ}(p / w)$ is a function characterized by circular symmetry and having unit intensity for $p\langle w$, and zero intensity for $\rho\rangle w$. The quantity $w$ defines the scaling of the near field radial intensity iistribution. The angle $\phi_{b}$ is the $1 / e^{2}$ half-width of the far field angular intensity distribution. Practically, it is the ratio of the $1 / \mathrm{e}^{2}$ focal spot radius to the focal length of an ideal lens or mirror. Alternatively, it is a bound on the ursertainty of direction of propagation in the collimated beam due to diffraction effects, For this $r_{c}$, on, the etendue of the collimated beam is given by ${ }_{b}=A_{b} \phi_{b}^{2}$.

Now, in order to estimate the area $A_{0}$ of a focal-plane distribution due to

"Other definitions of distribution width lead to different values of $\phi_{\mathrm{b}}$. For $\mathrm{ex}^{-}$ ample, $\phi_{b}=2.54$ gives the angular halfwidth of the circ far field which contains the same total, energy as is contained within the $1 / \mathrm{e}^{2}$ far field half angle of a Gaussian. a specific, near-ficid input to a focusing system to, say, the first zero or the $1 / \mathrm{e}^{2}$ point, one merely needs to know the convergence solid angla $\Omega$ of the focusing optic, and write

$$
A_{0}=\frac{\Phi_{0}}{\Omega}=\frac{\Phi_{b}}{\Omega} .
$$

In a paraxial ray problem where the fo: al region is a disk,

$$
A_{0}=\pi r_{0}^{2}=\frac{c^{2} \lambda^{2}}{\Omega}=\frac{4 c^{2} \lambda^{2} s_{\#}^{2}}{\pi}
$$

and

$$
r_{0}=\frac{2 c}{\pi} f_{H} \lambda .
$$

while, for example, in the problem of illuminating an entire hemisphere,

$$
\begin{aligned}
& A_{0}=2 \pi r_{0}^{2} \\
& r_{0}=\frac{\sqrt{2 c}}{\pi} f_{\#} \lambda .
\end{aligned}
$$

In any case, the definition for $f$-number given in Eq. (B3) is sufficiestly general to allow estimation of focal region diameters under appropriate definitions as the product of $f$-number and wavelength with errors which are typically $\pm 50 \%$. 


\section{Appendix C. \\ Proof of Equation (8)}

Referring to $\mathrm{Fig}$. 11 ; if $\beta$ is the firstiurface half-cone angle, the path of the intermediate ray in $(r, 2)$ coordinates is given by

$r_{I}(x, \rho)=\left(\frac{2 m}{1-m^{2}}\right) z-\left(\frac{1+m^{2}}{1-m^{2}}\right) \rho$,

where

$$
m=\tan \beta .
$$

The equation of the second reflective surface (orthogonal to the first in cross section) is

$$
r_{2}(z, \rho)=\frac{z_{0}-z}{n_{1}},
$$

where

$$
\mathrm{z}_{\mathrm{o}}=\left(\frac{1+\mathrm{m}^{2}}{2 \mathrm{~m}}\right) \mathrm{c} .
$$

The point of intersection of the intermediate ray with this surface obtained from Eqs. (C1) and (C3) is ( $r, z)$ where

$$
z=\left(\frac{1-m^{2}}{1+m^{2}}\right) z_{0}+m o
$$

so that

$r=\left(\frac{z_{0}-Z}{m}\right)=\left(\frac{2 m z_{o}}{1+m^{2}}\right)-\rho=c-\rho$. (C6)

Therefore, $d r=-d \rho$ as claimed in Eq. (8).

HLL/ bl/ Im:c 